05

\title{
Нелинейное преобразование магнитного шума в магнитоэлектрической структуре
}

\author{
(ㄱ Д.А. Бурдин, Ю.К. Фетисов, Д.В. Чашин, Н.А. Экономов
}

Московский технологический университет

E-mail: phantastic@mail.ru

Поступило в Редакцию 6 июня 2017 г.

Экспериментально обнаружен и исследован эффект преобразования спектра магнитного шума в планарной магнитоэлектрической структуре Metglasцирконат-титанат свинца-Metglas. Показано, что узкополосный шум с центральной частотой $f_{N}$ приводит к линейной генерации шумового электрического напряжения на частоте шума и нелинейной генерации шумового напряжения вблизи нулевых частот и в области удвоенной частоты шума. Эксперимент хорошо объясняется теорией смешения магнитных полей в магнитоэлектрических композитах с нелинейной зависимостью магнитострикции ферромагнетика от поля.

DOI: $10.21883 /$ PJTF.2017.18.45040.16905

Магнитоэлектрический (МЭ) эффект в композитных структурах, содержащих механически связанные ферромагнитные $(\Phi М)$ и пьезоэлектрические (ПЭ) слои, возникает в результате комбинации магнитострикции ФМ-слоя и пьезоэлектричества в ПЭ-слое из-за механической связи между слоями [1]. При этом нелинейная зависимость магнитострикции ФМ-слоя $\lambda$ от магнитного поля приводит к ряду нелинейных эффектов. Так, под действием гармонического поля накачки $h(f)$ и постоянного поля смещения $H$ структура генерирует электрическое напряжение $u(f)$ на частотах гармоник [2-6], под действием бигармонической накачки она генерирует напряжения с суммарной и разностной частотами $[7,8]$, обнаружена статическая деформация структур в переменном поле [9]. До сих пор нелинейные МЭ-эффекты в композитных структурах наблюдали только в условиях гармонической накачки. Вместе с тем на структуру может действовать и магнитный шум, который приведет к генерации шумового напряжения. В настоящей работе впервые исследовано преобразование спектра магнитного шума в нелинейной МЭ-структуре. 

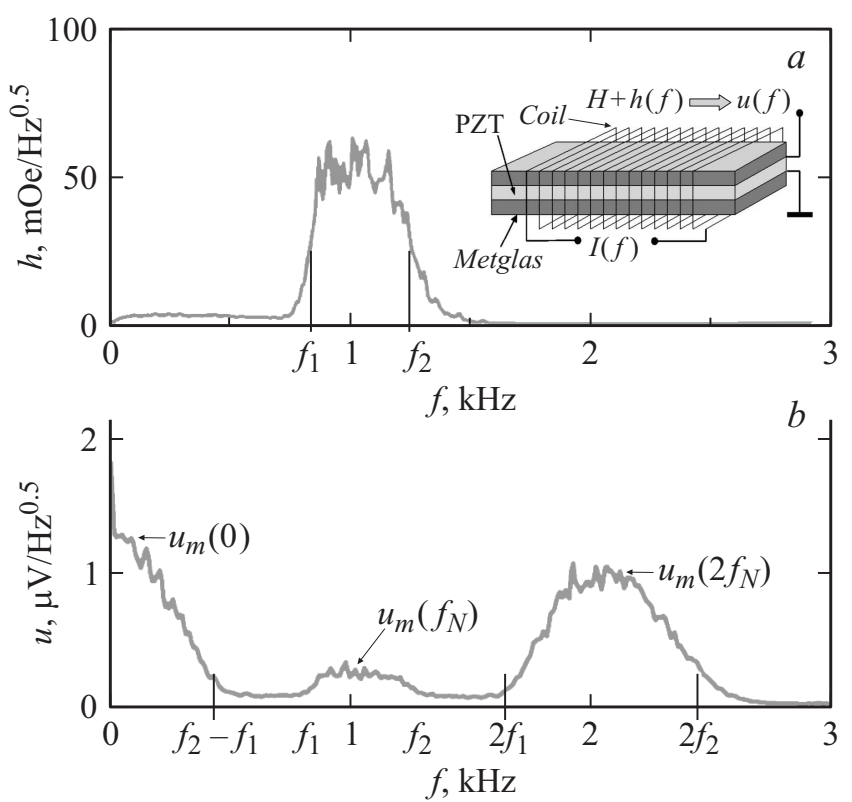

Рис. 1. Спектры магнитного шума $(a)$ и МЭ-напряжения $(b)$. На вставке схематично показана структура Metglas-PZT-Metglas.

В измерениях использовалась композитная структура, схематически изображенная на вставке к рис. 1,a. Структура содержала ПЭ-слой цирконата-титаната свинца $\mathrm{Pb}\left(\mathrm{Zr}_{0.52} \mathrm{Ti}_{0.48}\right) \mathrm{O}_{3}$ (PZT), расположенный между слоями аморфного сплава FeBSiC (Metglas), соединенными эпоксидным клеем. Пластина PZT размером $20 \times 10 \mathrm{~mm}$ и толщиной $100 \mu \mathrm{m}$ имела диэлектрическую проницаемость $\varepsilon=1750$ и пьезомодуль $d_{31}=175 \mathrm{pC} / \mathrm{m}$. Слои Metglas размером $20 \times 10 \mathrm{~mm}$ и толщиной $30 \mu \mathrm{m}$ имели магнитострикцию $\lambda_{S} \approx 21 \cdot 10^{-6}$ в полях насыщения $H_{S} \sim 60$ Ое. Структура была помещена в катушку, создающую магнитное поле накачки $h(f)$. Для создания накачки с шумовым спектром катушка подключалась к генератору шума Agilent 33210a, за которым располагались полосовой фильтр SR650 и широкополосный усилитель тока. Схема позволяла формировать поле с шумовым спектром примерно прямоугольной формы с центральной частотой $f_{N}=0.5-10 \mathrm{kHz}$, 
шириной полосы $\Delta=0.2-5 \mathrm{kHz}$, крутизной склонов $115 \mathrm{~dB} /$ oct и спектральной плотностью до $80 \mathrm{mOe} / \mathrm{Hz}^{0.5}$. Постоянное поле $H$, направленное вдоль оси структуры, создавали катушки Гельмгольца. Спектр частот накачки $h(f)$ и спектр частот МЭ-напряжения $u(f)$ регистрировались с помощью спектроанализатора SR770 при различных $h$ и $H$. Измерения проводились в нерезонансном режиме при частотах накачки меньше частот акустических резонансов структуры. Магнитострикция слоя Metglas $\lambda(H)$ измерялась тензометрическим методом при намагничивании образца вдоль длинной оси.

На рис. 1 показаны спектр частот магнитного шума $h(f)$ и типичный спектр частот генерируемого структурой МЭ-напряжения $u(f)$. Спектр частот шума имеет форму пика почти прямоугольной формы с центральной частотой $f_{N}=\left(f_{1}+f_{2}\right) / 2=1.04 \mathrm{kHz}$ и шириной полосы на половине высоты $\Delta=f_{2}-f_{1} \approx 0.43 \mathrm{kHz}$. В спектре напряжения четко видны три пика. Пик вблизи центральной частоты шума $f_{N}$ имеет ширину $\sim \Delta$, пик вблизи центральной частоты $2 f_{N}-$ ширину $\sim 2 \Delta$. Кроме того, в отличие от случая возбуждения структуры гармонической накачкой в спектре наблюдается шумоподобный пик шириной $\sim \Delta$ в области частот вблизи нуля. Высота пика сравнима с высотой шумоподобного пика вблизи частоты $2 f_{N}$. Механизм формирования составляющих спектра объяснен далее.

На рис 2, а показаны измеренные зависимости нормированных высот этих трех пиков от поля $H$ при спектральной плотности шума $h=10 \mathrm{mOe} /\left(\mathrm{Hz}^{0.5}\right)$. Видно, что высота пика $u_{m}\left(f_{N}\right)$ с центральной частотой $f_{N}$ линейно растет с увеличением $H$, достигает максимума при $H_{m} \approx 21.8 \mathrm{Oe}$, а затем монотонно спадает при дальнейшем увеличении $H$. Высоты пиков $u_{m}(0)$ и $u_{m}\left(2 f_{N}\right)$, расположенных в области малых частот и вблизи частоты $2 f_{N}$, максимальны в отсутствие поля $H \approx 0$, резко спадают почти до нуля при $H_{m}$, снова растут до локального максимума в поле $\sim 35$ Ое и затем монотонно уменьшаются с увеличением $H$. Кривые $u(f)$ и $u(0)$ в пределах точности измерений наложились друг на друга.

На рис. 2, $b$ точками показаны измеренные зависимости высот всех трех пиков от плотности шума $h$ при $H=0.5$ Ое. Штриховыми линиями представлены линейная и квадратичная аппроксимации экспериментальных данных. Видно, что высота пика $u_{m}\left(f_{N}\right)$ линейно растет с увеличением $h$. Высоты пиков $u_{m}(0)$ и $u_{m}\left(2 f_{N}\right)$ растут квадратично с

Письма в ЖТФ, 2017, том 43, вып. 18 

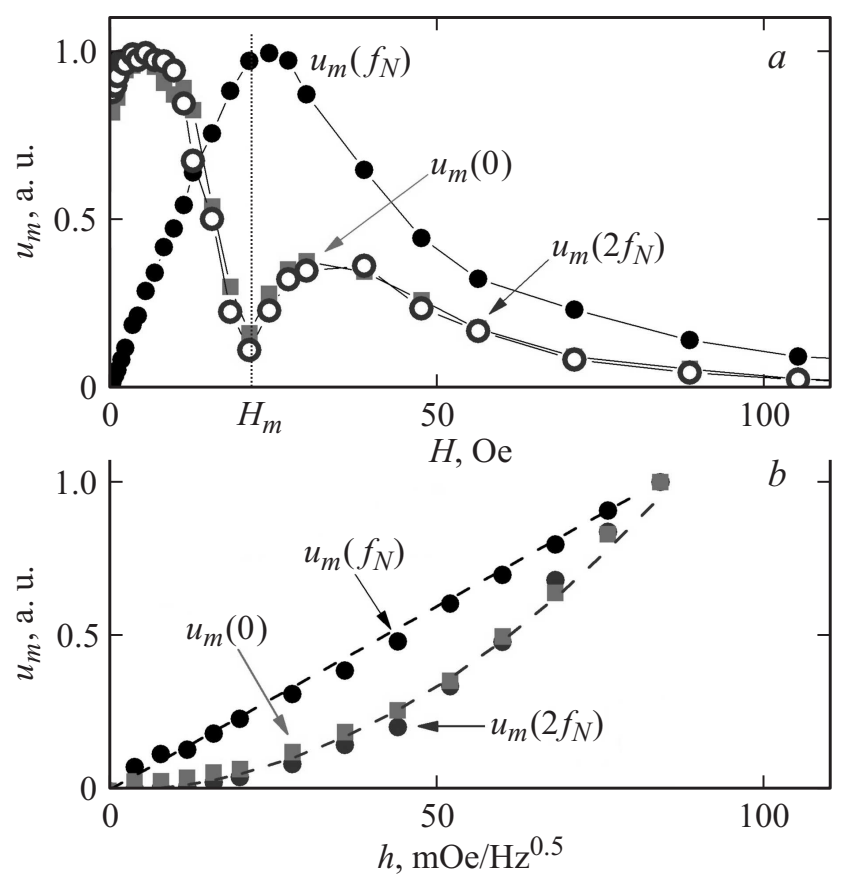

Рис. 2. Зависимости спектральной плотности компонент спектра от поля $H(a)$ и спектральной плотности шума $h(b)$. Штриховые линии - аппроксимация данных линейной и квадратичной функциями.

увеличением $h$. Кривые $u_{m}(0)$ и $u_{m}\left(2 f_{N}\right)$ в пределах точности измерений наложились друг на друга.

Для объяснения данных измерений используем модель смешения гармонических магнитных полей в композитной МЭ-структуре [8]. При действии на структуру поля $H$ в результате комбинации магнитострикции и пьезоэффекта ПЭ-слой структуры генерирует напряжение $u(H)=A d_{31} \lambda(H)$, где $A-$ постоянный коэффициент, зависящий только от размеров и материалов слоев структуры. Пусть поле накачки содержит две гармонические составляющие $H=H_{0}+h^{\prime} \cos \left(2 \pi f^{\prime} t\right)+h^{\prime \prime} \cos \left(2 \pi f^{\prime \prime} t\right)$ с частотами $f^{\prime}, f^{\prime \prime}$ и амплитудами $h^{\prime}, h^{\prime \prime}<H_{0}$. Разлагая $\lambda(H)$ в ряд Тейлора до членов второго 
порядка малости, подставляя разложение и поле $H$ в $u(H)$ и опуская постоянные слагаемые, получаем выражение для МЭ-напряжения

$$
\begin{aligned}
u & =A d_{31} \lambda^{(1)}\left[h^{\prime} \cos \left(2 \pi f^{\prime} t\right)+h^{\prime \prime} \cos \left(2 \pi f^{\prime \prime} t\right)\right] \\
& +(1 / 4) A d_{31} \lambda^{(2)}\left[h^{\prime} 2 \cos \left(4 \pi f^{\prime} t\right)+h^{\prime \prime} 2 \cos \left(4 \pi f^{\prime \prime} t\right)\right] \\
& +(1 / 2) A d_{31} \lambda^{(2)} \cos \left[2 \pi\left(f^{\prime} \pm f^{\prime \prime}\right) t\right] h^{\prime} h^{\prime \prime} .
\end{aligned}
$$

$\mathrm{B}(1) \lambda^{(1)}=\partial \lambda /\left.\partial H\right|_{H+0}$ и $\lambda^{(2)}=\partial^{2} \lambda /\left.H^{2}\right|_{H_{0}}$ - первая и вторая производные от магнитострикции по полю соответственно. Таким образом, структура должна генерировать гармоники напряжения на частотах накачки $f^{\prime}$ и $f^{\prime \prime}$, удвоенных частотах $2 f^{\prime}$ и $2 f^{\prime \prime}$, суммарной $f^{\prime}+f^{\prime \prime}$ и разностной $f^{\prime}-f^{\prime \prime}$ частотах.

Формирование спектра частот МЭ-напряжения под действием магнитного шума $h(f)$ со спектром прямоугольной формы в полосе частот $f_{1} f_{2}$ поясняет рис. 3,a. Нормированный спектр шума показан на рис. 3, $a$ штриховой линией. Каждая спектральная компонента шума генерирует напряжение со своей частотой, в результате чего в спектре МЭ-напряжения появляется шумовой пик с центральной частотой $f_{N}$ и спектральной плотностью $u=A d_{31} \lambda(H) h$. Кроме того, в спектре напряжения появляются гармоники с разностной $f^{\prime}-f^{\prime \prime}$ и суммарной $f^{\prime}+f^{\prime \prime}$ частотами, где частоты $f_{1} \leq f^{\prime}, f^{\prime \prime} \leq f_{2}$ лежат внутри полосы частот шума. Разностные гармоники дают шумовой пик напряжения шириной $\sim \Delta$, расположенный вблизи нулевых частот. Суммарные гармоники дают шумовой пик напряжения шириной $\sim 2 \Delta$ с центральной частотой $2 f_{N}$.

При расчете спектральной плотности напряжения $u(f)$ шумовых пиков необходимо учесть, что 1) в каждую составляющую спектра напряжения вносит вклад множество пар гармоник из спектра шума, поэтому нужно провести суммирование по спектру шума; 2) составляющие спектра магнитного шума некогерентны, поэтому следует складывать квадраты амплитуд гармоник напряжения, а затем вычислять спектральную плотность шумового напряжения.

Проведя вычисления, для спектральной плотности напряжения вблизи нулевых частот получаем

$$
u(0)=(1 / 2) A d_{31} \lambda^{(2)}(H) \sqrt{G(f)},
$$

где $G(f)=\int_{f_{1}}^{f_{2}-f} h^{2}\left(f^{\prime}\right) h^{2}\left(f+f^{\prime}\right) d f^{\prime}$.

Письма в ЖТФ, 2017, том 43, вып. 18 

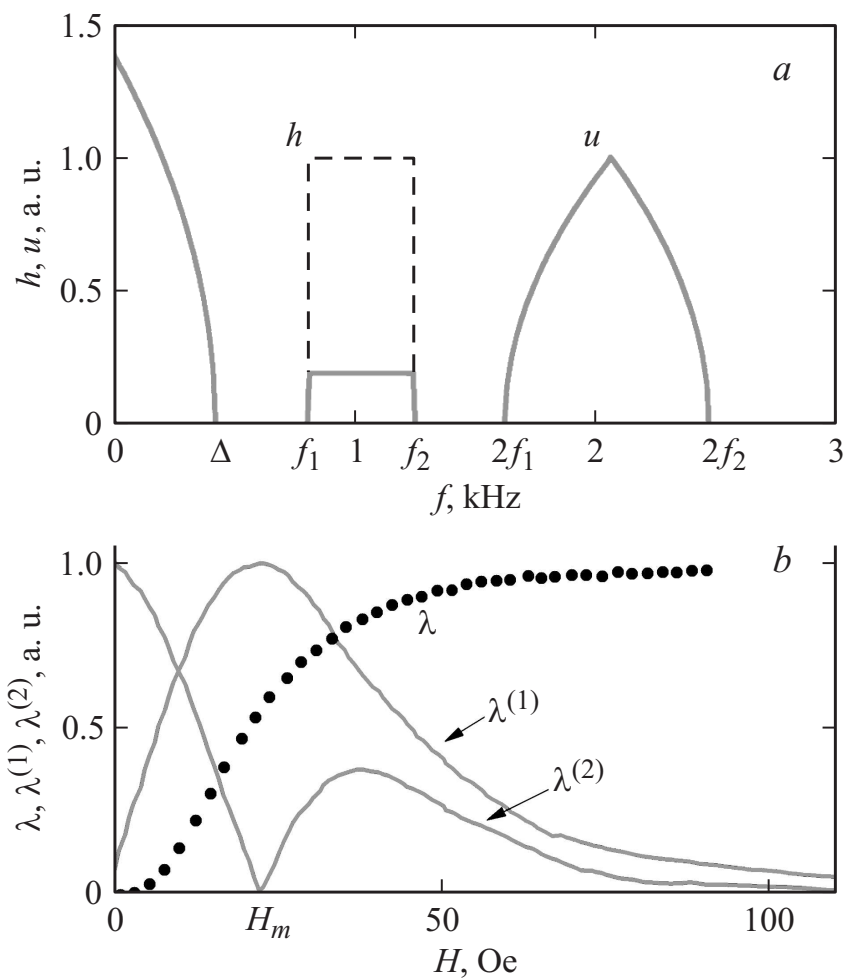

Рис. 3. $a-$ спектр частот магнитного шума (штриховая линия) и расчетный спектр частот МЭ-напряжения (сплошная линия). $b$ - зависимость магнитострикции $\lambda$ (точки) и ее производных $\lambda^{(1)}, \lambda^{(2)}$ (сплошные линии) от поля $H$ для слоя Metglas.

Для спектральной плотности напряжения вблизи частоты $2 f_{N}$ получаем

$$
u\left(2 f_{N}\right)=(1 / 2) A d_{31} \lambda^{(2)}(H) \sqrt{S(f)},
$$

где $S(f)=\int_{f_{1}}^{f_{2}} h^{2}\left(f^{\prime}\right) h^{2}\left(f-f^{\prime \prime}\right) d f^{\prime}$.

В случае магнитного шума со спектром прямоугольной формы $h\left(f^{\prime}\right)=h\left(f^{\prime \prime}\right)=h_{N}$ интеграл в (2) берется $G(f)=\sqrt{\Delta-f} h_{N}^{4}$, где 
$\Delta=f_{2}-f_{1}$ есть ширина спектра шума. Интеграл $S(f)$ в (3) - автокорреляционная функция, которая для спектра прямоугольной формы имеет вид треугольника с основанием шириной $2 \Delta$ и высотой $(1 / 2) h_{N}^{4}$.

Рассчитанный спектр частот напряжения от МЭ-структуры при возбуждении шумовой накачкой со спектром прямоугольной формы изображен на рис. $3, a$. Сравнение формы кривых на рис. $1, b$ и $3, a$ указывает на качественное совпадение эксперимента с расчетом. Различия связаны с непрямоугольной формой реального спектра магнитного шума и искажением формы шумового пика напряжения вблизи нулевых частот из-за токов утечки в PZT.

Вид зависимостей высот пиков в спектре МЭ-напряжения от постоянного магнитного поля $H$, согласно (1)-(3), определяется полевой зависимостью линейного $\lambda^{(1)}(H)$ и нелинейного $\lambda^{(2)}(H)$ пьезомагнитных модулей ФМ-слоя структуры. Для проверки была измерена кривая магнитострикции $\lambda(H)$ слоя Metglas (рис. $3, b)$ и с еe помощью численным методом найдены полевые зависимости производных от магнитострикции по полю. Максимальные величины производных равнялись $\quad \lambda_{m}^{(1)} \approx 7.5 \cdot 10^{-7} \mathrm{Oe}^{-1}, \quad \lambda_{m}^{(2)}(H=0) \approx 4 \cdot 10^{-8} \mathrm{Oe}^{-2}$. Для сравнения кривые на рис. $3, b$ нормированы. Хорошее совпадение формы измеренных кривых на рис. 2, $a$ и рассчитанных кривых на рис. $3, b$ подтверждает справедливость теории.

Из (1)-(3) следует, что высота шумового пика напряжения с центральной частотой $u_{m}\left(f_{N}\right)$ должна линейно зависеть от спектральной плотности шума $h_{N}$, а высота пиков напряжения $u_{m}(0)$ и $u_{m}\left(2 f_{N}\right)-$ квадратично. Штриховыми линиями на рис. $2, b$ показаны соответственно линейная и квадратичная аппроксимации экспериментальных данных. Видно, что расчет хорошо описывает зависимость МЭ-напряжения от спектральной плотности магнитного шума.

Таким образом, в работе обнаружен и исследован эффект преобразования спектра магнитного шума в планарной МЭ-структуре Metglas-PZT-Metglas. Показано, что узкополосный шум со спектром прямоугольной формы и центральной частотой $f_{N} \approx 1 \mathrm{kHz}$ приводит к линейной генерации шумового напряжения на частоте шума $f_{N}$ и нелинейной генерации шумового напряжения вблизи нулевых частот и в области удвоенной частоты $2 f_{N}$. Форма пиков спектральной плотности шумового напряжения линейной и нелинейной компонент спектра, а также полевая и амплитудная зависимости напряжения описываются

Письма в ЖТФ, 2017, том 43, вып. 18 
теорией смешения магнитных полей в МЭ-структурах с нелинейной зависимостью магнитострикции от поля. Результаты исследований могут оказаться полезными при разработке высокочувствительных МЭдатчиков магнитных полей.

Работа поддержана Российским фондом фундаментальных исследований (грант № 16-29-14017) и Министерством образования и науки РФ (проектная часть госзадания № 3.5350.2017/ВУ).

\section{Список литературы}

[1] Bichurin M.I., Petrov V.M. Srinivasan G. // Phys. Rev. B. 2003. V. 68. P. 054402.

[2] Kamentsev K.E., Fetisov Y.K., Srinivasan G. // Appl. Phys. Lett. 2006. V. 89. P. 142510.

[3] Zhang W., Yin G., Cao J., Bai J., Wei F. // Appl. Phys. Lett. 2012. V. 100. P. 032903.

[4] Fetisov L.Y., Fetisov Y.K., Sreenivasulu G., Srinivasan G. // J. Appl. Phys. 2013. V. 113. P. 116101.

[5] Лалетин В.М., Филиппов Д.А., Фирсова Т.Ю. // Письма в ЖТФ. 2014. Т. 40. B. 6. C. 11.

[6] Филиппов Д.А., Лалетин В.М., Фирсова Т.О. // ФТТ. 2014. Т. 56. В. 5. С. 944.

[7] Petrie J., Wiehland D., Gray D., Mandal S., Sreenivasulu G., Srinivasan G., Edelstein A.S. // J. Appl. Phys. 2011. V. 110. P. 124506.

[8] Burdin D.A., Chashin D.V., Ekonomov N.A., Fetisov L.Y., Fetisov Y.K., Sreenivasulu G., Srinivasan G. // J. Magn. Magn. Mater. 2014. V. 358-359. P. 98.

[9] Burdin D.A., Chashin D.V., Ekonomov N.A., Fetisov Y.K. // J. Magn. Magn. Mater. 2016. V. 406. P. 217. 